Elena García-González, Ricardo González-Tarancón, Maite Aramendía and Luis Rello*

\title{
Analytical interference by monoclonal immunoglobulins on the direct bilirubin AU Beckman Coulter assay: the benefit of unsuspected diagnosis from spurious results
}

DOI 10.1515/cclm-2015-0608

Received June 28, 2015; accepted November 11, 2015

\section{Abstract}

Background: Monoclonal (M) components can interfere with the direct bilirubin (D-Bil) assay on the AU Beckman Coulter instrumentation and produce spurious results, such as D-Bil values greater than total bilirubin (T-Bil) or very low/negative D-Bil values. If properly detected, this interference may uncover undiagnosed patients with monoclonal gammopathy (MG).

Methods: We investigated the interference rate on the D-Bil AU assay in serum samples known to contain M proteins along with their isotype and described the protocol set up in our laboratory to help with the diagnosis of MG based on D-Bil spurious results as first indication.

Results: During a period of 4 years, $15.4 \%$ (345 of 2235) of serum samples containing $\mathrm{M}$ immunoglobulins produced erroneous D-Bil results, although no clear relationship between the magnitude or isotype of the M component and interference could be found. In total 22 new patients were diagnosed with MG based on the analytical artefact with the D-Bil as first indication.

Conclusions: The D-Bil interference from MG on the Beckman AU analysers needs to be made known to laboratories in order to prevent clinical confusion and/or additional workup to explain the origin of anomalous results. Although this information may not add to the management of existing patients with serum paraproteins, it can benefit patients that have not been diagnosed with MG by

*Corresponding author: Luis Rello, Department of Clinical Biochemistry, "Miguel Servet” University Hospital, Paseo Isabel La Católica 1-3, 50009, Zaragoza, Spain, Phone: +34 976765544, Fax: +34976765543, E-mail: Irello@salud.aragon.es

Elena García-González and Ricardo González-Tarancón: Department of Clinical Biochemistry, “Miguel Servet” University Hospital, Zaragoza, Spain

Maite Aramendía: Centro Universitario de la Defensa-Academia General Militar de Zaragoza, Zaragoza, Spain triggering follow up testing to determine if M components are present.

Keywords: direct bilirubin; interference; monoclonal component; monoclonal gammopathy; paraprotein.

\section{Introduction}

It is well reported in literature that monoclonal (M) components (or paraproteins) may interfere with an extensive range of analytical techniques [1, 2], routine chemistry methods among them [3, 4]. Specifically, the total bilirubin (T-Bil) assay with the Roche platforms $[5,6]$ and the direct bilirubin (D-Bil) method with the AU Beckman Coulter platforms [6-8] have been shown to occasionally produce spurious results in the presence of $M$ proteins of high concentration due to abnormal reactions with measurement reagents [9]. Efforts to detect such interfered results are not systematically carried out in clinical laboratories, even though this would be interesting for different reasons.

For instance, elevated T-Bil values with low (that is, within reference population intervals) D-Bil levels are clinically possible as, for example, in haemolytic anaemia or liver disease. As a result, interfered samples providing spuriously high $\mathrm{T}$-Bil results might be incorrectly suspected of presenting one of the previously mentioned medical conditions (even if the patient is known to have a paraprotein), which may trigger unnecessary additional diagnostic procedures to investigate the cause of this high T-Bil value. This problem could be significantly minimised if a warning flag to inform of an anomalous reaction data would be implemented by the manufacturers of automatic analysers, as recently described for a T-Bil assay [9].

As for D-Bil analyses, M protein interference may not even be detected if the T-Bil is normal and, therefore, the D-Bil is not quantified. However when both tests are analysed simultaneously and the D-Bil level is higher than the T-Bil or negative D-Bil values are obtained, some 
investigation should be carried out to verify if an M protein interference is the cause of the spurious results. In 2005, Nauti et al. [7] first described examples in three patients with IgG kappa paraproteins whose D-Bil values measured on the AU analysers (previously Olympus Diagnostics) were an order of magnitude greater than the corresponding T-Bil values. Later, Yang et al. [6] demonstrated that this method-specific interference could be more frequent than suspected as they reported a percentage of interference as high as $44 \%$ in sera containing $M$ components, with approximately $16 \%$ of samples with $\mathrm{D}$-Bil values greater than the T-Bil and $28 \%$ of samples with negative D-Bil values. This last possibility is more likely to be overlooked in everyday work of clinical laboratories. In fact, M protein interference may cause not only negative, but also low D-Bil values, which might not be flagged by the system and pass unnoticed. To detect such interference, Yang et al. monitored manually the photometric reaction of each measurement as, unfortunately, the AU instruments do not allow raising flags based on the kind of anomalous reaction data described to be produced by this interference [6]. More recently, Song et al. [8] reported that the D-Bil AU assay may be affected not only by monoclonal but also by polyclonal immunoglobulins.

While it is important to know that some assays can be interfered by paraproteins and lead to misdiagnosis [10], an even more important effect could be drawn from detection of all $\mathrm{M}$ protein interferences in T- or D-Bil results. In fact, it is possible from such anomalous results to identify undiagnosed patients with MG. This fact has somewhat been suggested in the publications cited above but it has not been systematically studied. For example, Seimiya et al. [9], after introducing a warning flag in the T-Bil reaction data in their BM series JEOL analyser, detected seven samples having M proteins (from a total of 30,731 samples over a period of 2 months). This anomalous reaction was the first indication for conducting electrophoresis and immunofixation tests in three of these cases. Since approximately $3 \%$ of people over 50 years of age have a MGUS (monoclonal gammopathy of undetermined significance) [11] and its progression to malignancy is approximately $1 \%$ per year [12], one can speculate that the number of samples containing paraproteins which can interfere in some of the chemistry assays may be greater than reported, as the study by Seimiya et al. [9] seems to indicate.

Considering all of the above, the aim of this study was twofold. The first aim was to investigate, over a large period of time ( 4 years), the patients being seen at the Haematology Department of the Hospital Universitario Miguel Servet (HUMS) possibly having M proteins, for the magnitude of the effect of M proteins on the D-Bil assay along with the isotype of the M component. Secondly, and considering that at our Centre there were no limitations on clinicians ordering simultaneously T-Bil and D-Bil tests, we systematically studied all samples having a D-Bil value greater than the T-Bil or, alternatively, those with suspiciously low D-Bil values, for the presence of an unsuspected MG.

\section{Material and methods}

\section{Samples and definition of interference in the D-Bil assay}

We reviewed the D-Bil and T-Bil results for 2235 serum samples (from June 2010 to May 2014) from 617 different patients studied at the Haematology Department of the HUMS suspected of, or under study for, having $\mathrm{M}$ proteins confirmed by immunofixation, for falsely increased (above the T-Bil) or suspiciously low D-Bil concentrations. When an initial suspiciously low D-Bil result was obtained, paraprotein interference was considered to be demonstrated when repetition of analysis produced markedly variable results, as proposed by Yang et al. [6]

Alternatively, during the same period of time, 27 serum samples collected from patients not suspected to have MG and whose D-Bil values were anomalous (greater than the T-Bil or suspiciously low) were investigated to determine the presence of a M protein by IgG, IgA and IgM quantification, protein electrophoresis and immunofixation.

In all cases, only samples free of hemolysis, icterus, and lipemia (determined by routine photometric measurements) were included in the study. As expected over such a long period of time, several samples from the same patients were obtained at different times, especially in the case of those followed up at the Haematology Department, so it was possible to evaluate the relationship, within the same patients, between the M protein magnitude and the interference. Due to the high subjectivity of the evaluation of the M concentration by densitometry (uncertainty on how to set the limits of the M peak and inclusion of the polyclonal background on which it sits), it was preferred to use the immunoglobulin concentration corresponding to the $\mathrm{M}$ isotype in the study. Also, for the sake of simplicity, the small number of samples with free light chains components, heavy chains components, or double $\mathrm{M}$ components were excluded from evaluation.

Written informed consent was obtained from all patients followed up at the Haematology Department and also from the additional 27 patients further studied to assess the presence of a MG. The study was approved by the Hospital Universitario Miguel Servet Ethics Committee.

\section{Instrumentation}

D-Bil, T-Bil and total protein (TP) concentrations were measured on the AU analysers (Beckman Coulter, Brea, CA, USA). Details of the D-Bil AU assay fundamentals along with the cause of interference by $M$ proteins can be found elsewhere $[6,8]$. IgG, IgA, and IgM 
concentrations were determined by nephelometry on the Immage 800 apparatus (Beckman Coulter). Serum protein electrophoresis and immunofixation were carried out with the HYDRASYS system (Sebia, Norcross, GA, USA). The M protein concentration was calculated by multiplying the relative density of the $\mathrm{M}$ band provided by electrophoresis by the TP concentration.

\section{Results}

\section{Interference rate in samples known to contain an M component}

From a total of 2235 samples from 617 different patients studied at the Haematology Department and having a serum M protein confirmed by immunofixation, 137 (6.1\%) produced D-Bil results greater than the T-Bil. Suspiciously low D-Bil results were obtained for 237 samples, although only in 208 cases (that is, $9.3 \%$ of the total number of samples) interference by an anomalous reaction could be demonstrated by obtaining highly variable results after analysis repetition (four replicates in two consecutive runs of the same sample aliquot). Table 1 shows several examples of replicate results obtained with those samples. As can be seen, very low values maintained an acceptable concordance between replicates, whereas in suspicious samples an initial very low or even negative value was followed by highly variable replicates, some of them well above the T-Bil result.

Thus, the total interference rate calculated was $15.4 \%$ (345 of 2235). If samples with low M concentration were excluded from this evaluation (that is, only M components whose concentration was $>20 \mathrm{~g} / \mathrm{L}$ for IgG, $15 \mathrm{~g} / \mathrm{L}$ for IgA and $10 \mathrm{~g} / \mathrm{L}$ for IgM were considered) the percentage of samples interfered was $32.9 \%, 283$ of 861 samples, $14.1 \%$ with a D-Bil greater than the T-Bil and $18.8 \%$ with spuriously low results, respectively.

With respect to the $\mathrm{M}$ concentration and isotype of the samples interfered, Table 2 displays the number of samples reviewed and the percentage of interference calculated for each $\mathrm{M}$ isotype. This is shown considering all the samples with the same isotype as a whole, or separated into arbitrary ranges of concentration for each isotype (normal-slightly incremented, high and overtly high). As seen from this Table, the higher the M concentration, the greater the possibility of interference.

Finally, for samples from the same patients obtained at different times and which exhibited interference on at least one occasion, this seemed to occur when the $\mathrm{M}$ concentration tended to be higher, but similar M concentrations at other instances did not produce overtly anomalous D-Bil results. These details are shown for selected patients in Figure 1.

\section{Unsuspected diagnosis of monoclonal gammopathy by D-Bil spurious results}

The exact number of D-Bil determinations carried out in our laboratory from adult patients not known to contain $\mathrm{M}$ proteins in the 4 years of study is difficult to evaluate with precision, but a figure of 300,000 will not be far from reality. After excluding the main causes of interference in the T-Bil and D-Bil assays (as intense hemolysis or lipemia, for example), a total of 27 samples from adult patients without previous results indicating the existence

Table 1: Examples of the variability obtained for replicate D-Bil analyses of samples that provided an initial suspiciously low D-Bil result.

\begin{tabular}{|c|c|c|c|c|c|c|c|}
\hline & \multirow[t]{2}{*}{ Total bilirubin, $\mathrm{mg} / \mathrm{L}$} & \multirow[b]{2}{*}{ Replicate 1} & \multirow[b]{2}{*}{ Replicate 2} & \multirow[b]{2}{*}{ Replicate 3} & \multicolumn{2}{|c|}{ Direct bilirubin, $\mathrm{mg} / \mathrm{L}^{\mathrm{a}}$} & \multirow[t]{2}{*}{ Conclusion } \\
\hline & & & & & Replicate 4 & $\mathbf{S D}^{\mathrm{b}}$ & \\
\hline Sample 1 & 4.4 & -0.3 & 1.9 & -28.5 & -5.9 & 11.3 & Interfered \\
\hline Sample 2 & 3.1 & -0.1 & -0.2 & 0.5 & 0.1 & 0.3 & True low \\
\hline Sample 3 & 4.2 & 0.3 & 0.6 & 0.6 & 0.7 & 0.2 & True low \\
\hline Sample 4 & 5.0 & -4.3 & 0.9 & 4.9 & 7.9 & 5.3 & Interfered \\
\hline Sample 5 & 6.7 & 0.1 & 4.1 & -5.0 & 1.5 & 3.8 & interfered \\
\hline Sample 6 & 3.5 & -0.6 & 0.5 & 0.5 & 0.3 & 0.5 & true low \\
\hline Sample 7 & 5.6 & -1.3 & -2.6 & 0.8 & 1.7 & 2.0 & interfered \\
\hline Sample 8 & 4.6 & -0.6 & 6.2 & -4.5 & 8.6 & 6.0 & interfered \\
\hline
\end{tabular}

aThe limit of detection of the D-Bil AU assay is $0.14 \mathrm{mg} / \mathrm{L}$, so measurements below this value are informed in laboratory reports as $<0.14$. Here we have preferred to represent the real value obtained in each replicate to illustrate the variability of each sample whose initial value

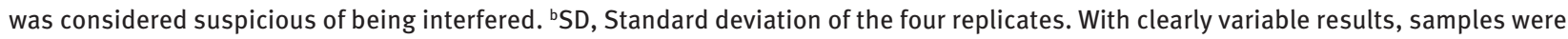
classified as "interfered" and further assayed for the existence of paraproteins. Samples with fairly homogeneous results were classified as "true low". 
Table 2: Interference rate on the D-Bil AU assay with samples from patients followed up at the Haematology Department of the HUMS, known to contain paraproteins and with a serum $M$ component confirmed by immunofixation.

\begin{tabular}{|c|c|c|c|c|c|}
\hline \multirow[t]{2}{*}{ Isotype } & \multicolumn{3}{|c|}{ Number (\%) of samples with $M$ component } & \multicolumn{2}{|c|}{ n (\%) of samples interfered } \\
\hline & n (\% total samples) & Ig concentration, g/L (arbitrary range) & $\mathrm{n}$ (\% same isotype) & $\%$ same isotype & $\%$ same concentration \\
\hline \multirow[t]{3}{*}{ IgG kappa } & $989(44.3 \%)$ & $<20$ (normal-slightly high) & $565(57.1 \%)$ & $169(17.1 \%)$ & $29(5.1 \%)$ \\
\hline & & 20-40 (high) & $358(36.2 \%)$ & & $100(27.9 \%)$ \\
\hline & & >40 (overtly high) & $66(6.7 \%)$ & & $40(60.6 \%)$ \\
\hline \multirow[t]{3}{*}{ IgG lambda } & $458(20.5 \%)$ & $<20$ (normal-slightly high) & $319(70.7 \%)$ & $77(16.8 \%)$ & $21(6.6 \%)$ \\
\hline & & 20-40 (high) & $126(27.5 \%)$ & & $46(36.5 \%)$ \\
\hline & & >40 (overtly high) & $13(2.8 \%)$ & & $10(76.9 \%)$ \\
\hline \multirow[t]{3}{*}{ IgA kappa } & $247(11.1 \%)$ & <15 (normal-slightly high) & $152(61.5 \%)$ & $17(6.9 \%)$ & $2(1.3 \%)$ \\
\hline & & $15-30$ (high) & $63(25.5 \%)$ & & $8(12.7 \%)$ \\
\hline & & >30 (overtly high) & $32(13.0 \%)$ & & $7(21.9 \%)$ \\
\hline \multirow[t]{3}{*}{ IgA lambda } & $160(7.2 \%)$ & <15 (normal-slightly high) & 99 (61.9\%) & $33(20.6 \%)$ & $6(6.1 \%)$ \\
\hline & & $15-30$ (high) & $46(28.8 \%)$ & & $15(32.6 \%)$ \\
\hline & & $>30$ (overtly high) & $15(9.4 \%)$ & & $12(80.0 \%)$ \\
\hline \multirow[t]{3}{*}{ IgM kappa } & $295(13.2 \%)$ & $<10$ (normal-slightly high) & $193(65.4 \%)$ & $47(15.9 \%)$ & $3(1.6 \%)$ \\
\hline & & 10-20 (high) & $49(16.6 \%)$ & & $12(24.5 \%)$ \\
\hline & & $>20$ (overtly high) & $53(18.0 \%)$ & & $32(60.4 \%)$ \\
\hline \multirow[t]{3}{*}{ IgM lambda } & $86(3.8 \%)$ & $<10$ (normal-slightly high) & $46(53.5 \%)$ & $2(2.3 \%)$ & $1(2.2 \%)$ \\
\hline & & 10-20 (high) & $22(25.6 \%)$ & & $1(4.5 \%)$ \\
\hline & & $>20$ (overtly high) & $18(20.9 \%)$ & & $0(0.0 \%)$ \\
\hline \multirow[t]{3}{*}{ Total } & $2235(100 \%)$ & Normal-slightly high & $1374(61.5 \%)$ & $345(15.4 \%)$ & $62(4.5 \%)$ \\
\hline & & high & $664(29.7 \%)$ & & $182(27.4 \%)$ \\
\hline & & overtly high & $197(8.8 \%)$ & & $101(51.3 \%)$ \\
\hline
\end{tabular}

They are classified regarding paraprotein isotype and, within each isotype, stratified in arbitrary ranges of normal-slightly high, high and overtly high concentration of the corresponding immunoglobulin isotype.

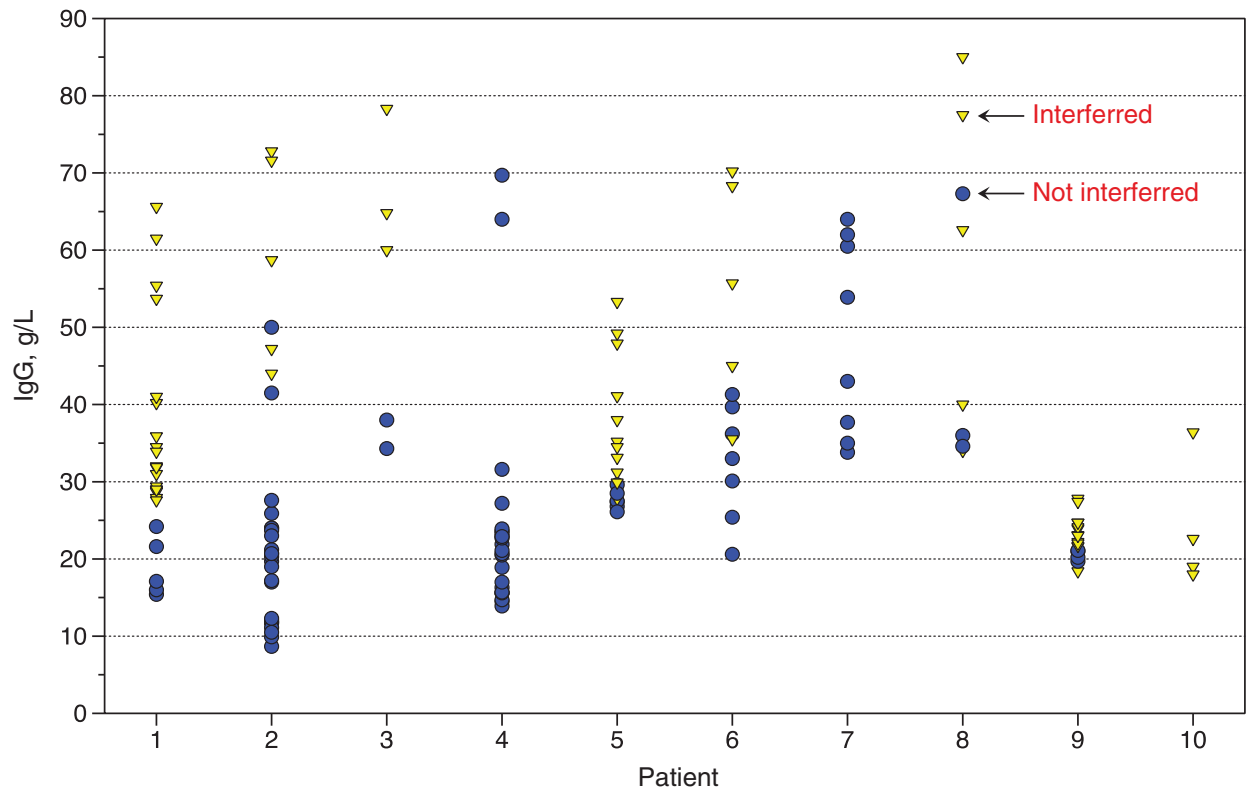

Figure 1: Relationship between M component concentration and interference in the D-Bil AU Beckman Coulter assay in samples from 10 selected patients with IgG kappa paraproteins collected at different times over a period of 4 years.

of an $\mathrm{M}$ component exhibited anomalous D-Bil results. Therefore, the presence of paraproteins was subsequently investigated in these 27 cases. In 22 of these patients an
M component was identified and the patients were transferred to the Haematology Department where the MG was formally diagnosed and classified. Table 3 shows 
Table 3: Main analytical and clinical characteristics of the patients diagnosed with monoclonal gammopathy as a consequence of spurious direct bilirubin results.

\begin{tabular}{|c|c|c|c|c|c|c|c|c|}
\hline $\begin{array}{l}\text { Assay, units } \\
\text { (reference range) }\end{array}$ & $\begin{array}{l}\text { Direct bilirubin, } \\
\mathrm{mg} / \mathrm{L}(0.0-2.0)\end{array}$ & $\begin{array}{r}\text { Total bilirubin, } \\
\mathrm{mg} / \mathrm{L}(3.0-12.0)\end{array}$ & $\begin{array}{r}\operatorname{IgA}, \mathrm{g} / \mathrm{L} \\
(0.8-3.5)\end{array}$ & $\begin{array}{r}\operatorname{IgG}, \mathrm{g} / \mathrm{L} \\
(6.5-16.0)\end{array}$ & $\begin{array}{r}\operatorname{IgM}, \mathrm{g} / \mathrm{L} \\
(0.5-3.0)\end{array}$ & Isotype & $\begin{array}{r}\text { M com., } \\
\mathrm{g} / \mathrm{L}\end{array}$ & Pathology \\
\hline Patient 1 & 4.3 & 4.0 & 3.4 & 31 & 0.7 & Polyclonal & & \\
\hline Patient 2 & 14.7 & 4.2 & 0.6 & 12.4 & 0.4 & IgG lambda & 9.1 & MGUS \\
\hline Patient 3 & 4.4 & 3.2 & 1.8 & 17.5 & 1.5 & IgG kappa & 12.0 & MGUS \\
\hline Patient 4 & $<0.14$ & 3.8 & 6.0 & 23 & 1.8 & Polyclonal & & \\
\hline Patient 5 & 4.7 & 4.0 & 0.3 & 7.3 & 22 & IgM Kappa & 13.3 & WM \\
\hline Patient 6 & 9.1 & 3.6 & 1.1 & 18.7 & 0.3 & IgG Kappa & 14.2 & MM \\
\hline Patient 7 & 50.8 & 7.9 & 0.3 & 30 & 0.2 & IgG Карра & 21 & MM \\
\hline Patient 8 & 16.2 & 6.2 & 0.8 & 28 & 0.4 & IgG Lambda & 21 & MM \\
\hline Patient 9 & 56.4 & 4.9 & 0.3 & 61 & 0.1 & IgG Lambda & 40 & MM \\
\hline Patient 10 & 4.8 & 4.1 & 1.3 & 28 & 0.4 & IgG Kappa & 13.6 & MM \\
\hline Patient 11 & 4.1 & 3.8 & 5.4 & 33 & 1.3 & Polyclonal & & \\
\hline Patient 12 & 13.9 & 8.4 & 1.4 & 19.2 & 0.5 & IgG Kappa & 8.0 & MGUS \\
\hline Patient 13 & 11.2 & 4.3 & 1.7 & 3.7 & 71 & IgM Карра & 53 & WM \\
\hline Patient 14 & $<0.14$ & 3.1 & 0.2 & 33 & 0.2 & IgG Lambda & 26 & MM \\
\hline Patient 15 & $<0.14$ & 4.5 & 1.8 & 22 & 1.7 & IgG Kappa & 15.8 & MGUS \\
\hline Patient 16 & 10.8 & 3.0 & 1.4 & 25 & 0.6 & IgG Карра & 18.0 & MGUS \\
\hline Patient 17 & 7.0 & 2.9 & 25 & 2.2 & 0.5 & IgA Lambda & 30 & MM \\
\hline Patient 18 & 5.4 & 4.1 & 1.2 & 6.5 & 20 & IgM Карра & 13.1 & WM \\
\hline Patient 19 & $<0.14$ & 5.9 & 35 & 9.9 & 0.3 & IgA Lambda & 22 & MM \\
\hline Patient 20 & 11.8 & 3.4 & 8.5 & 36 & 2.8 & Polyclonal & & \\
\hline Patient 21 & $<0.14$ & 2.8 & 4.3 & 42 & 1.2 & Polyclonal & & \\
\hline Patient 22 & $<0.14$ & 3.4 & 0.4 & 80 & 0.1 & IgG Карра & 59 & MM \\
\hline Patient 23 & $<0.14$ & 4.0 & 0.3 & 72 & 0.1 & IgG Карра & 48 & MM \\
\hline Patient 24 & $<0.14$ & 5.3 & 79 & 3.3 & 0.1 & IgA Lambda & 49 & MM \\
\hline Patient 25 & 5.2 & 5.1 & 2.9 & 15.3 & 0.7 & IgG Kappa & 12.9 & MGUS \\
\hline Patient 26 & $<0.14$ & 3.0 & 0.1 & 100 & 0.1 & IgG Kappa & 60 & MM \\
\hline Patient 27 & 27.4 & 4.4 & 0.1 & 53 & 0.1 & IgG Kappa & 38 & MM \\
\hline
\end{tabular}

aMGUS, Monoclonal gammopathy of undetermined significance; WM, Waldenström's macroglobulinemia; MM, myeloma multiple.

analytical and clinically relevant information from these patients. The existence of an M component could not be demonstrated for the remaining five samples, but they showed elevated polyclonal immunoglobulins, supporting the conclusions presented in the work by Song et al. [8] that polyclonal immunoglobulins may also interfere the D-Bil AU assay.

Interestingly, in those 22 patients newly diagnosed with MG because of a D-Bil spurious result as first indication, most of them $(68 \%, 15$ of 22$)$ exhibited an initial D-Bil result greater than the T-Bil, and only in seven cases (32\%) an initial suspiciously low result was obtained.

\section{Discussion}

Literature contains numerous examples of unsuspected diagnosis of MG arising from spurious results for most chemistry tests [4], or even from serum indexes [13], but these findings have been often isolated and not systematically studied. The protocol that we describe in this publication was established in collaboration with the Haematology Department of our hospital, and is to be used for detecting the analytical interference and utilising this information to diagnose patients with MG prior to the appearance of symptoms.

As the AU instrumentation does not allow to automatically raise flags based on photometric readings, we implemented a protocol where the Laboratory Information System (LIS) flagged all suspicious D-Bil results. In this sense, D-Bil values above the corresponding T-Bil value or below $0.4 \mathrm{mg} / \mathrm{L}$ (a value that, in some cases, can be considered suspiciously low) were flagged. Those flagged results are invalidated by the LIS and must be reviewed one by one by laboratory specialists. In the case of low D-Bil results, the specialist must decide whether repeating the measurement or approving it, based on the patient clinical and laboratory history being easily accessible from the LIS. In that way, most D-Bil spurious results are confirmed to be from patients already known to have paraproteins (or from samples with intense hemolysis or 
lipemia) and only 2-3 samples on a daily basis (from a total of $900 \mathrm{D}$-Bil determinations per day) are subjected to analysis repetition (see Table 1). As all this information is freely accessible from the same computer, it only requires some minutes of additional work. Finally, the samples not known to contain paraproteins and suspected of interference are subsequently analyzed in our laboratory to verify its presence, by means of immunoglobulin quantification, protein electrophoresis and, if appropriate, immunofixation. When a paraprotein is diagnosed, the Haematology Department is notified so they can commence the patient clinical evaluation and management. Most patients diagnosed with MG in this way (Table 3) were being evaluated for other pathological conditions such as pneumonia, bone pain disorders and some of them were totally asymptomatic at the time of the diagnosis. Therefore, in these cases, the protocol developed has helped to diagnose MG at least several months earlier, which represents a clear benefit for these patients.

Interestingly, our study also seems to indicate that some patients may have been missed by the protocol set up in our laboratory or that, unfortunately, it is not always correctly applied, since the percentage of samples unknown to contain paraproteins with an initial suspiciously low D-Bil value (32\%, 7 out of 22 ) is lower than those further studied as a consequence of a D-Bil greater than the T-Bil. This is in contradiction with our own results obtained with the patients known to have an $\mathrm{M}$ protein (69\% of samples interfered presented an initial low D-Bil value) and also with the percentages reported in other works (64\% and 55\% in references 6 and 8, respectively). This clearly highlights the weakness of only employing the LIS for detecting anomalous results, and reinforces the idea that present instrumentation should be supplied with an adequate flagging system to monitor all reactions carried out. Only in this way, all the samples interfered in the D-Bil AU assay would be detected, even those that provide completely reasonable results (see, e.g. some of the replicates in Table 1).

\section{Conclusions}

The interference on the D-Bil AU assay produced by paraproteins is patient dependent and therefore, specific to the particular M protein. In addition, for the majority of patients who exhibited interference, this only occurs with high $\mathrm{M}$ concentrations.

For patients already known to have serum paraproteins, this knowledge could only serve to avoid additional workup, but for patients not diagnosed with MG it should call for additional testing to assess the presence of $\mathrm{M}$ components.

The discovering of this interference based on the initial D-Bil result obtained with each sample is clearly unsatisfactory, as even interfered reactions could provide a reasonable value by mere chance, so that they will not be flagged by the LIS and, therefore, will be skipped from revision. We do encourage the manufacturer to include a warning of this interference in the D-Bil technical information to promote its knowledge to all new users and, most importantly, to develop a reaction data monitoring system so that all anomalous D-Bil measurements can be properly detected.

Author contributions: All the authors have accepted responsibility for the entire content of this submitted manuscript and approved submission.

Research funding: None declared.

Employment or leadership: None declared.

Honorarium: None declared.

Competing interests: The funding organisation(s) played no role in the study design; in the collection, analysis, and interpretation of data; in the writing of the report; or in the decision to submit the report for publication.

\section{References}

1. Kroll MH, Elin RJ. Interference with clinical laboratory analyses. Clin Chem 1994;40:1996-2005.

2. Tate J, Ward G. Interferences in immunoassay. Clin Biochem Rev 2004;25:105-20.

3. Bakker AJ, Mücke M. Gammopathy interference in clinical chemistry assays: mechanisms, detection and prevention. Clin Chem Lab Med 2007;45:1240-3.

4. Dalal BI, Brigden ML. Factitious biochemical measurements resulting from hematologic conditions. Am J Clin Pathol 2009;131:195-204.

5. Pantanowitz L, Horowitz GL, Upalakalin JN, Beckwith BA. Artifactual hyperbilirubinemia due to paraprotein interference. Arch Pathol Lab Med 2003;127:55-9.

6. Yang Y, Howanitz PJ, Howanitz JH, Gorfajn H, Wong K. Paraproteins are a common cause of interferences with automated chemistry methods. Arch Pathol Lab Med 2008;132:217-23.

7. Nauti A, Barassi A, Merlini G, d'Eril GV. Paraprotein interference in an assay of conjugated bilirubin. Clin Chem 2005;51:1076-7.

8. Song L, Kelly KA, Butch AW. Monoclonal and polyclonal immunoglobulin interference in a conjugated bilirubin assay. Arch Pathol Lab Med 2014;138:950-4.

9. Seimiya M, Suzuki Y, Yoshida T, Sawabe Y, Matsushita K, Nomura F. The abnormal reaction data-detecting function of the automated biochemical analyzer was useful to prevent erroneous total-bilirubin measurement and to identify monoclonal proteins. Clin Chim Acta 2015;441:44-6. 
10. Jelinek AG, Bachmann LM. Unexpected test results in a patient with multiple myeloma. Clin Chem 2014;60:1375-8.

11. Wadhera RK, Rajkumar SV. Prevalence of monoclonal gammopathy of undetermined significance: a systematic review. Mayo Clin Proc 2010;85:933-42.
12. Kyle RA, Rajkumar SV. Monoclonal gammopathies of undetermined significance. Best Pract Res Clin Haematol 2005;18:689-707.

13. Monk C, Wallage M, Wassell J, Whiteway A, James J, Beetham R. A monoclonal protein identified by an anomalous lipaemia index. Ann Clin Biochem 2009;46:250-2. 